\title{
Method to compute the longitudinal coupling impedance of an iris in a vacuum chamber
}

\author{
D. Davino \\ Dipartimento di Ingegneria Elettrica, Università di Napoli Federico II, Via Claudio 21, I-80125 Napoli, Italy \\ and INFN Sezione di Napoli, Complesso Universitario di Monte S. Angelo, I-80126 Napoli, Italy \\ G. Miano \\ Dipartimento di Ingegneria Elettrica, Università di Napoli Federico II, Via Claudio 21, I-80125 Napoli, Italy \\ and INFN Sezione di Napoli, Complesso Universitario di Monte S. Angelo, I-80126 Napoli, Italy \\ G. Panariello \\ Dipartimento di Ingegneria Elettronica, Università di Napoli Federico II, Via Claudio 21, I-80125 Napoli, Italy \\ L. Verolino \\ Dipartimento di Ingegneria Elettrica, Università di Napoli Federico II, Via Claudio 21, I-80125 Napoli, Italy \\ and INFN Sezione di Napoli, Complesso Universitario di Monte S. Angelo, I-80126 Napoli, Italy
}

(Received 23 September 1998; published 5 April 1999)

\begin{abstract}
This paper deals with the evaluation of the longitudinal coupling impedance due to a charged particle traveling along the axis of an iris in a circular waveguide. The solution of the electromagnetic problem is given in terms of a dual series expansion, and some interesting developments of this technique are presented in this paper. [S1098-4402(99)00032-4]
\end{abstract}

PACS numbers: 41.75.-i, 41.20.-q, 29.27.-a

\section{INTRODUCTION}

Let us consider a charged particle traveling at constant velocity $v$ in the positive direction of the $z$ axis along the symmetry axis of a vacuum chamber, namely a perfectly conducting cylindrical waveguide (pipe) of radius $b$. The symmetry axis coincides with the $z$ axis of a cylindrical coordinates' system. A circular iris of radius $a$ is placed in correspondence to the abscissa $z=0$ in the chamber, and the particle will come across the iris at $t=0$.

The effects of the field radiated from the iris on the longitudinal motion of the beam can be described in the frequency domain through the longitudinal coupling impedance, a global parameter defined by the integral $[1,2]$

$$
\begin{aligned}
Z_{\|}(k)=-\frac{1}{q} \int_{-\infty}^{+\infty} & {\left[E_{z}(r=0, z)\right.} \\
& \left.-E_{z}^{0}(r=0, z)\right] e^{j k z / \beta} d z,
\end{aligned}
$$

where $E_{z}^{0}(r=0, z)$ represents the longitudinal component of the electric field in the vacuum chamber without iris and $\beta=v / c$.

Field matching is one of the most known techniques to calculate the longitudinal impedance. Kheifets [3] proposed this method to study step junctions and simple cavities. Some phenomena of slow or relative convergence [4] due to the singularity of the electromagnetic field can occur using the field matching technique. The radiated field has been recently evaluated by means of an integral formulation by Gluckstern [5] for both slim and thick irises, but only for particles traveling at the speed of light.

Today the scientific community of accelerator physics is projecting and constructing hadron machines [6], working with values of $\beta$ less than 1 . Therefore an accurate and robust method able to evaluate the radiated field, and hence the longitudinal impedance, is needed for any value of $\beta$.

The aim of this paper is to evaluate the longitudinal coupling impedance of a circular iris for any value of $\beta$ in a wide range of the aspect ratio $w=a / b$ (the ratio between the radius of the iris and the one of the vacuum chamber) by means of a new technique, based on dual series [7]. The electromagnetic field due to the induced currents for each wave number $k$ can be expanded according to the TM modes of the waveguide $e_{n}(r)$, and, in order to satisfy boundary conditions, the expansion coefficients have to obey the following system of coupled equations:

$$
\begin{array}{ll}
\sum_{n=1}^{\infty} \frac{k b}{\sqrt{(k b)^{2}-x_{n}^{2}}} c_{n} e_{n}(r)=0, & 0 \leq r \leq a, \\
\sum_{n=1}^{\infty} c_{n} e_{n}(r)=-\frac{\beta \sqrt{\pi}}{q \zeta} E_{r}^{0}(r, 0), & a \leq r \leq b,
\end{array}
$$

where $E_{r}^{0}(r, 0)$ is the field of the particle traveling in an infinite uniform waveguide, and the meaning of adopted symbols will be fully clarified in the following. The first equation in the system (2) is obtained by enforcing the continuity of the azimuthal component of the magnetic field in the hole of the iris, and the second one is obtained by imposing that the radial component of the electric field vanishes on the metallic region of the iris.

We propose a new technique, suggested for different problems by Sneddon [7] and Tranter [8], and already elaborated for a continuous case of a dual integral equation system by ourselves [9], which enables us to take into account the right edge behavior (given by the Meixner 
condition [10]) by means of an adequate expansion of the unknown coefficients $c_{n}$ and, therefore, can give a high rate of convergence. Thus the solution of our problem is reduced to the solution of a system of complex algebraic equations of low order (typically less than 10).

It is also given an approximated formula valid for all values of $\beta$ which reduces the computational load in the high frequency limit.

\section{REPRESENTATION OF THE FIELDS}

In this section we give a representation of the electromagnetic fields useful to solve the proposed radiation problem.

The diffraction mechanism can be described by the superposition of the field traveling with the charge itself and the field radiated by the iris, which has a traveling wave character. Accordingly, we can represent the fields as the superposition of two terms

$$
\mathbf{E}=\mathbf{E}^{0}+\mathbf{E}^{S}, \quad \mathbf{H}=\mathbf{H}^{0}+\mathbf{H}^{S},
$$

a term generated by the charge in an infinite waveguide $\left(\mathbf{E}^{0}, \mathbf{H}^{0}\right)$ without the iris, and a term created by the presence of the iris $\left(\mathbf{E}^{S}, \mathbf{H}^{S}\right)$, which together have to satisfy boundary conditions. It is worth noting that, owing to the symmetry of the problem, induced currents on the iris are in the radial direction and therefore there is a TM propagation with radial and longitudinal components for the electric field and only the azimuthal one for the magnetic field.

A charge moving with uniform velocity in a vacuum chamber radiates only because of the inhomogeneities present near its trajectory, as the circular iris, located at $z=0$, shown in Fig. 1. The radiation process is essentially due to the diffraction of the primary field of the particle in correspondence to the iris. The field created by the charge in the presence of the iris will interact with the charge itself so that, together with the radiation, we should find a decrease of the particle velocity. Such a radiation problem is very difficult to solve and therefore a simplifying assumption will be made: We shall suppose that the charge moves at constant velocity during its fly. The constant velocity can be imagined as being maintained by an external source. The result will be a good approximation provided that the velocity of the charge does not change significantly during the interaction

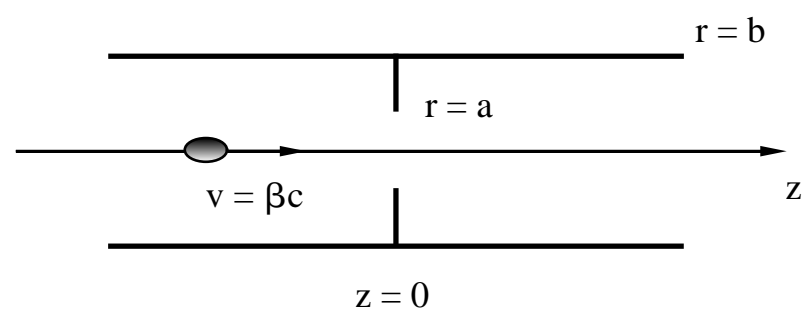

FIG. 1. Geometry of the problem. with the iris. This assumption can be considered to be realistic when dealing with ultrarelativistic charges.

The field of an ultrarelativistic charge is essentially confined within an angular region of aperture $\approx 1 / \gamma$, where

$$
\gamma=\frac{1}{\sqrt{1-\beta^{2}}}
$$

is the Lorentz factor, namely the energy of the charge expressed in rest mass units. As long as the charge is far from the iris, it barely perceives the presence of the discontinuity. Its image charges follow it along the lateral surface of the cylinder. In this situation, which persists up to quite small distances of the charge from the junction, little radiation is expected. Only when the iris is seen by the charge within the narrow $(\approx 1 / \gamma)$ cone of its field will the image charges experience a sudden change of their motion since they are released and start moving in the radial direction up to the cylinder of larger radius: This process lasts for the time of passage of the charge through the hole and it is the main reason for radiation. The more relativistic is the charge, the shorter is the radiation time and the wider is the spectrum of radiation.

As is well known, a particle of charge $q$, traveling at a constant velocity $v=\beta c$ along the axis of a circular waveguide of radius $b$, sustains the TM electromagnetic field

$$
\begin{aligned}
& E_{r}^{0}(r, z)=\frac{q \zeta \kappa}{2 \pi \beta}\left[K_{1}(\kappa r)+\frac{I_{1}(\kappa r)}{I_{0}(\kappa b)} K_{0}(\kappa b)\right] e^{-j k z / \beta}, \\
& E_{z}^{0}(r, z)=\frac{j q \zeta \kappa}{2 \pi \beta \gamma}\left[K_{0}(\kappa r)-\frac{I_{0}(\kappa r)}{I_{0}(\kappa b)} K_{0}(\kappa b)\right] e^{-j k z / \beta}, \\
& H_{\varphi}^{0}(r, z)=\frac{q \kappa}{2 \pi}\left[K_{1}(\kappa r)+\frac{I_{1}(\kappa r)}{I_{0}(\kappa b)} K_{0}(\kappa b)\right] e^{-j k z / \beta},
\end{aligned}
$$

where $\kappa=k / \beta \gamma$ and $\zeta$ is the characteristic impedance of the medium filling the waveguide. If the particle moves at the speed of light, previous fields become independent by the radius of the waveguide and simplify as

$$
\begin{gathered}
E_{r}^{0}(r, z)=\frac{q \zeta}{2 \pi r} e^{-j k z / \beta}, \quad E_{z}^{0}(r, z)=0, \\
H_{\varphi}^{0}(r, z)=\frac{q}{2 \pi r} e^{-j k z / \beta} .
\end{gathered}
$$

In order to solve the electromagnetic problem, we considered Maxwell equations related to the scattered field for a TM azimuthal symmetric propagating field

$$
\begin{gathered}
\frac{1}{r} \frac{\partial}{\partial r}\left(r E_{r}\right)+\frac{\partial E_{z}}{\partial z}=0, \quad \frac{\partial E_{r}}{\partial z}-\frac{\partial E_{z}}{\partial r}=-j k \zeta H_{\varphi}, \\
\frac{\partial H_{\varphi}}{\partial r}=-j \frac{k}{\zeta} E_{r}, \quad \frac{1}{r} \frac{\partial}{\partial r}\left(r H_{\varphi}\right)=j \frac{k}{\zeta} E_{z},
\end{gathered}
$$

where $k=\omega \sqrt{\varepsilon \mu}=\omega / c$ represents the wave number and $\zeta=\sqrt{\mu / \varepsilon}$ is the characteristic impedance of the medium filling the waveguide. 
According to the modal theory of the waveguides, the transverse electric field can be represented as

$$
E_{r}(r, z)= \begin{cases}E_{r}^{0}(r, z)+\frac{q \zeta}{\beta \sqrt{\pi}} \sum_{n=1}^{\infty} c_{n}^{-} e_{n}(r) \exp \left[j z \sqrt{(k b)^{2}-x_{n}^{2}}\right], & z<0, \\ E_{r}^{0}(r, z)+\frac{q \zeta}{\beta \sqrt{\pi}} \sum_{n=1}^{\infty} c_{n}^{+} e_{n}(r) \exp \left[-j z \sqrt{(k b)^{2}-x_{n}^{2}}\right], & z>0,\end{cases}
$$

whereas the magnetic field is given by

$$
H_{\varphi}(r, z)= \begin{cases}H_{\varphi}^{0}(r, z)-\frac{q k}{\beta \sqrt{\pi}} \sum_{n=1}^{\infty} c_{n}^{-} e_{n}(r) \frac{\exp \left[j z \sqrt{(k b)^{2}-x_{n}^{2}}\right]}{\sqrt{(k b)^{2}-x_{n}^{2}}}, \quad z<0, \\ H_{\varphi}^{0}(r, z)+\frac{q k}{\beta \sqrt{\pi}} \sum_{n=1}^{\infty} c_{n}^{+} e_{n}(r) \frac{\exp \left[-j z \sqrt{(k b)^{2}-x_{n}^{2}}\right]}{\sqrt{(k b)^{2}-x_{n}^{2}}}, \quad z>0 .\end{cases}
$$

It is worth noting that the value of the longitudinal propagation constant (choice of the branch cut in a complex plane) is different for propagative and nonpropagative modes according to the following scheme:

$$
\sqrt{k^{2}-\left(x_{n} / b\right)^{2}}= \begin{cases}\sqrt{k^{2}-\left(x_{n} / b\right)^{2}}, & \text { if }|k|>x_{n} / b \text { (propagative modes), } \\ -j \sqrt{\left(x_{n} / b\right)^{2}-k^{2}}, & \text { if }|k|<x_{n} / b \text { (cutoff modes) }\end{cases}
$$

$x_{n}$ being the $n$th zero of the Bessel function $J_{0}(x)$, namely $J_{0}\left(x_{m}\right)=0$ within $m=1,2,3, \ldots$; the expansion functions $[11,12]$

$$
e_{m}(r)=\frac{J_{1}\left(x_{m} r / b\right)}{b \sqrt{\pi} J_{1}\left(x_{m}\right)}
$$

are orthonormal in the transverse section of the waveguide

$$
\iint_{S} e_{n}(r) e_{m}(r) d S= \begin{cases}1, & n=m \\ 0, & n \neq m\end{cases}
$$

Expansions (5) and (6) automatically verify the radiation condition at infinity $|z| \rightarrow \infty$, and the adopted normalizations imply dimensionless expansion coefficients $c_{n}^{ \pm}$.

\section{STATEMENT OF THE PROBLEM}

In order to solve our boundary value problem for Maxwell equations, we have to impose the continuity of the radial component of the electric field and of the azimuthal component of the magnetic field on the hole of the iris, and we require that the radial component of the electric field vanishes on the metallic region of the iris.
Moreover, since the induced current is orthogonal to the edge, the Meixner (or edge) condition [10] requires that the components of the electric field diverge as $d^{-1 / 2}, d$ being the distance from the edge, in the hole region.

First of all, introducing the notations in the transverse section $z=0$

$$
\begin{gathered}
S_{a}=\{r: 0 \leq r \leq a\}, \quad S_{b}=\{r: 0 \leq r \leq b\}, \\
S_{b}-S_{a}=\{r: a \leq r \leq b\},
\end{gathered}
$$

we start by imposing the continuity of the radial component of the electric field on the transverse section containing the iris, namely

$$
E_{r}\left(r, 0^{+}\right)=E_{r}\left(r, 0^{-}\right), \quad r \in S_{b},
$$

which enables us to verify immediately that

$$
c_{n}^{-}=c_{n}^{+}, \quad \forall n .
$$

This conclusion enables us to rewrite the field expansions in the more compact form

$$
\begin{gathered}
\frac{2 \pi b}{q \zeta}\left[E_{r}(r, z)-E_{r}^{0}(r, z)\right]=\frac{2}{\beta} \sum_{n=1}^{\infty} c_{n} \frac{J_{1}\left(x_{n} r / b\right)}{J_{1}\left(x_{n}\right)} \exp \left[-j|z| \sqrt{(k b)^{2}-x_{n}^{2}}\right], \\
\frac{2 \pi b}{q}\left[H_{\varphi}(r, z)-H_{\varphi}^{0}(r, z)\right]=\frac{2 k b}{\beta} \operatorname{sgn}(z) \sum_{n=1}^{\infty} c_{n} \frac{J_{1}\left(x_{n} r / b\right)}{J_{1}\left(x_{n}\right)} \frac{\exp \left[-j|z| \sqrt{(k b)^{2}-x_{n}^{2}}\right]}{\sqrt{(k b)^{2}-x_{n}^{2}}},
\end{gathered}
$$

where, for the sake of shortness, we indicated two dimensionless quantities related to the radial component of the electric field and the azimuthal component of the magnetic field that will be the object of numerical evaluation and discussion below (Fig. 2).

The second condition we have to impose states that the radial component of the electric field vanishes on the metallic region of the iris, formally
$E_{r}(r, 0)=0, \quad r \in S_{b}-S_{a}$,

or, using the expansion (13), we can explicitly write

$$
\sum_{n=1}^{\infty} c_{n} e_{n}(r)=-\frac{\beta \sqrt{\pi}}{q \zeta} E_{r}^{0}(r, z), \quad a \leq r \leq b .
$$



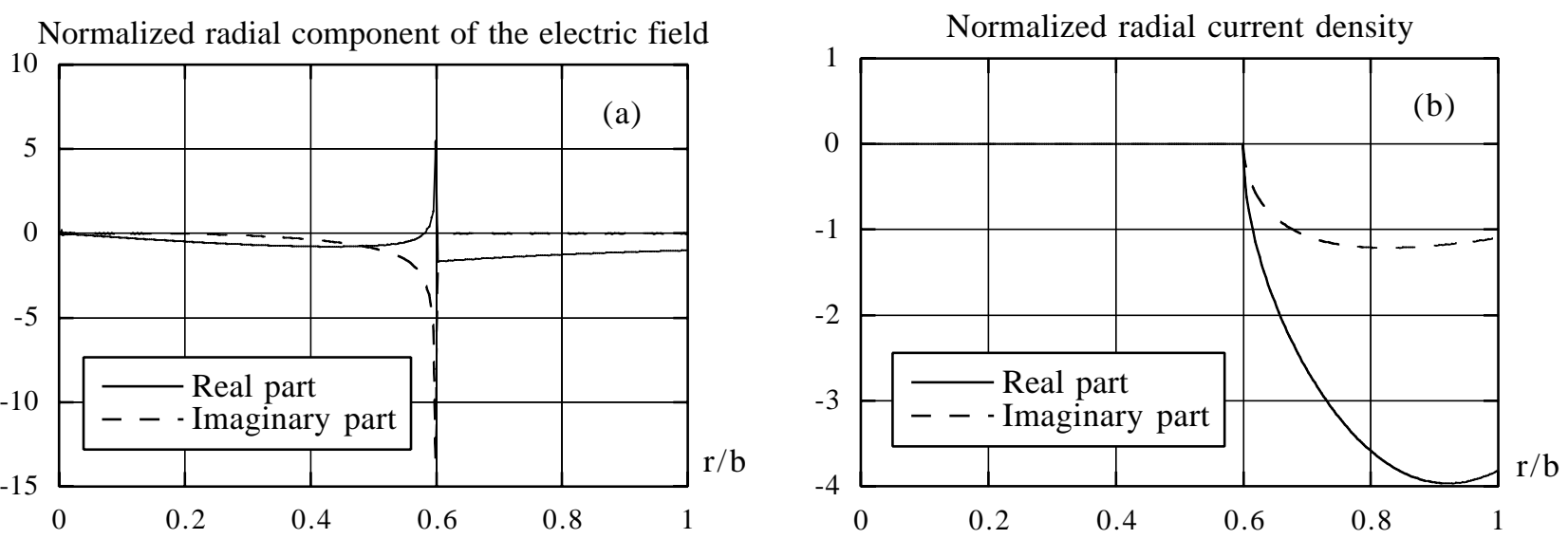

FIG. 2. The radial component of the electric field normalized at $2 \pi b / \zeta q$ at the iris (a) and the radial current density normalized at $2 \pi b / q$ (b) in the case $k b=5, w=3 / 5$, and $\beta=1$

The last requirement we do is the continuity of the azimuthal component of the magnetic field on the hole defining the iris, that is

$$
H_{\varphi}\left(r, 0^{+}\right)=H_{\varphi}\left(r, 0^{-}\right), \quad r \in S_{a},
$$

which can be put in the explicit form

$$
H_{\varphi}^{0}(r, z)+\frac{q k}{\beta \sqrt{\pi}} \sum_{n=1}^{\infty} \frac{c_{n} e_{n}(r)}{\sqrt{(k b)^{2}-x_{n}^{2}}}=H_{\varphi}^{0}(r, z)-\frac{q k}{\beta \sqrt{\pi}} \sum_{n=1}^{\infty} \frac{c_{n} e_{n}(r)}{\sqrt{(k b)^{2}-x_{n}^{2}}}, \quad r \in S_{a},
$$

or in a dimensionless form

$$
\sum_{n=1}^{\infty} c_{n} e_{n}(r) \frac{k b}{\sqrt{(k b)^{2}-x_{n}^{2}}}=0, \quad 0 \leq r \leq a .
$$

Therefore we can state that our electromagnetic problem is summarized by two series equations, namely the system made by Eqs. (2), which is a system of dual series, valid in complementary regions.

Finally, it is interesting to investigate the surface current density induced by the particle during its fly $J_{r}(r)=$ $H_{\varphi}\left(r, 0^{+}\right)-H_{\varphi}\left(r, 0^{-}\right)$which has only the radial component, given by

$$
\frac{2 \pi b}{q} J_{r}(r)=\frac{4}{\beta} \sum_{n=1}^{\infty} c_{n} \frac{J_{1}\left(x_{n} r / b\right)}{J_{1}\left(x_{n}\right)} \frac{k b}{\sqrt{(k b)^{2}-x_{n}^{2}}} .
$$

It is apparent that the boundary condition (16), representing the continuity of the magnetic field on the hole, implies that

$$
J_{r}(r)=0, \quad 0 \leq r \leq a
$$

$$
\frac{2 \pi b}{q \zeta} E_{r}(r, z)=\frac{2 \pi b}{q \zeta} E_{r}^{0}(r, z)[u(r)-u(r-a)]+\frac{2}{\beta} \sum_{n=1}^{\infty} d_{n} \frac{J_{1}\left(x_{n} r / b\right)}{J_{1}\left(x_{n}\right)} \exp \left[-j|z| \sqrt{(k b)^{2}-x_{n}^{2}}\right],
$$

where the second addendum still contains the electromagnetic field singularity for $r=a$, so the system (2) becomes

\section{SOLUTION OF THE PROBLEM}

Dual series equation systems occur, in general, in mixed boundary value problems where the metallic region is finite (or in the dual problem). The basic method used to solve a system of this type is to find an adequate representation of the unknown field which is skilled to satisfy one of the two equations and to transform the other one into an easily manageable system of algebraic equations.

In order to reduce the system (2) to the so-called canonical form for the dual series systems, we introduce the new unknown coefficients $d_{n}=c_{n}+b_{n}$, where we define

$$
b_{m}=\frac{\beta \sqrt{\pi}}{q \zeta} \iint_{S_{b}-S_{a}} E_{r}^{0}(r, 0) e_{m}(r) d S
$$

namely the expansion coefficients of the radial component of the primary electric field

$$
E_{r}^{0}(r, 0)[u(r-a)-u(r-b)]=\frac{q \zeta}{\beta \sqrt{\pi}} \sum_{n=1}^{\infty} b_{n} e_{n}(r),
$$

(evaluated in Appendix A). Therefore the total electric field can be written in the form 


$$
\begin{array}{ll}
\sum_{n=1}^{\infty} d_{n} e_{n}(r) \frac{k b}{\sqrt{(k b)^{2}-x_{n}^{2}}}=\sum_{n=1}^{\infty} b_{n} e_{n}(r) \frac{k b}{\sqrt{(k b)^{2}-x_{n}^{2}}}, & 0 \leq r \leq a, \\
\sum_{n=1}^{\infty} d_{n} e_{n}(r)=0, & a \leq r \leq b,
\end{array}
$$

In order to expand the radial component of the electric field factorizing the edge singularity according to the Meixner condition [10], let us consider the class of functions

$$
F_{m}(r)= \begin{cases}\frac{\sqrt{2} \Gamma(m)}{\Gamma(m-1 / 2)} \frac{r}{\sqrt{a^{2}-r^{2}}} P_{m-1}^{(1,-1 / 2)}\left[1-2(r / a)^{2}\right], & 0 \leq r \leq a \\ 0, & a \leq r \leq b\end{cases}
$$

where $m$ is a positive integer and $P_{n}^{(\alpha, \beta)}(x)$ are Jacobi polynomials [11]. Of course, the completeness of the modal functions (8) enables us to represent the function $F_{m}(r)$ by using the modal functions $e_{n}(r)$ (details are given in Appendix B),

$$
F_{m}(r)=\sum_{n=1}^{\infty}\left[2 w \sqrt{\frac{\pi a b}{x_{n}}} \frac{J_{2 m-1 / 2}\left(w x_{n}\right)}{J_{1}\left(x_{n}\right)}\right] e_{n}(r) .
$$

So, if we call $u(r)$ the unit step function and we assume the following representation of the unknown coefficients $d_{n}$,

$$
d_{n}=\frac{2 w}{\sqrt{x_{n}} J_{1}\left(x_{n}\right)} \sum_{n=1}^{\infty} f_{m} J_{2 m-1 / 2}\left(w x_{n}\right),
$$

we obtain the following representation of the electric field

$$
\frac{2 \pi b}{q \zeta} E_{r}(r, z)=\frac{2 \pi b}{q \zeta} E_{r}^{0}(r, z)[u(r)-u(r-a)]+\frac{4 w}{\beta} \sum_{m=1}^{\infty} f_{m}\left[\sum_{n=1}^{\infty} \frac{J_{2 m-1 / 2}\left(w x_{n}\right) J_{1}\left(x_{n} r / b\right)}{\sqrt{x_{n}} J_{1}^{2}\left(x_{n}\right)}\right],
$$

and using the relation (21) it is

$$
E_{r}(r, 0)=\left\{E_{r}^{0}(r, 0)+\frac{\zeta q}{\pi b \beta} \sqrt{\frac{2}{w}} \frac{r}{\sqrt{a^{2}-r^{2}}} \sum_{m=1}^{\infty} \frac{\Gamma(m) f_{m}}{\Gamma(m-1 / 2)} P_{m-1}^{(1,-1 / 2)}\left[1-2(r / a)^{2}\right]\right\}[u(r)-u(r-a)],
$$

which is skilled to reproduce the discontinuity at $r=a$.

Furthermore, the second equation of the system (20) is automatically satisfied in force of the identity (B6). On the other hand, coefficients $f_{m}$ have to be chosen so that $d_{n}$ also satisfies the first equation of the system, which becomes

$$
\begin{gathered}
\sum_{m=1}^{\infty} f_{m}\left[\sum_{n=1}^{\infty} \frac{J_{2 m-1 / 2}\left(w x_{n}\right) J_{1}\left(x_{n} r / b\right)}{\sqrt{x_{n}} J_{1}^{2}\left(x_{n}\right)} \frac{k b}{\sqrt{(k b)^{2}-x_{n}^{2}}}\right] \\
=\sum_{n=1}^{\infty} \frac{b_{n}}{2 w} \frac{J_{1}\left(x_{n} r / b\right)}{J_{1}^{2}\left(x_{n}\right)} \frac{k b}{\sqrt{(k b)^{2}-x_{n}^{2}}} .
\end{gathered}
$$

Multiplying both members of the relation (24) by

$$
\frac{r P_{s-1}^{(1,-1 / 2)}\left[1-2(r / a)^{2}\right]}{\sqrt{a^{2}-r^{2}}},
$$

integrating on the interval $(a, b)$, and interchanging the order of integration and series, we obtain the infinite set of linear (complex) equations

$$
\sum_{m=1}^{\infty} A_{s m} f_{m}=h_{s}, \quad s=1,2, \ldots,
$$

where the generic element of the coefficients matrix is defined by

$$
A_{s m}=\sum_{n=1}^{\infty} \frac{J_{2 m-1 / 2}\left(w x_{n}\right) J_{2 s-1 / 2}\left(w x_{n}\right)}{x_{n} J_{1}^{2}\left(x_{n}\right)} \frac{k b}{\sqrt{(k b)^{2}-x_{n}^{2}}},
$$

and the component of the free term vector is given by

$$
h_{s}=\frac{1}{2 w} \sum_{n=1}^{\infty} b_{n} \frac{J_{2 s-1 / 2}\left(w x_{n}\right)}{\sqrt{x_{n}} J_{1}\left(x_{n}\right)} \frac{k b}{\sqrt{(k b)^{2}-x_{n}^{2}}} .
$$

It is worth noting that the matrix $\mathbf{A}$ is symmetric and diagonally dominant as shown in Fig. 3, where the absolute value of the elements of the main diagonal and the first parallel has been drawn. In the low frequency limit (Fig. 3a) we can say that this behavior is confirmed, whereas when the frequency grows up (Fig. 3b) this property is less manifest, and the matrix becomes "full." Figure 4 shows that even at high frequency $(k b=10)$ ten expansions coefficients $f_{N}$ are sufficient.

\section{LONGITUDINAL COUPLING IMPEDANCE}

The global parameter taking into account the interaction between the bunch of charged particles and the surrounding structure is the longitudinal coupling impedance, defined by the integral (1). Since from the Maxwell equation $\nabla \times \overrightarrow{\mathbf{H}}=j \omega \varepsilon \overrightarrow{\mathbf{E}}$, the longitudinal component of the electric field can be written as

$$
E_{z}=-j \frac{\zeta}{k}(\nabla \times \overrightarrow{\mathbf{H}})_{z}=-j \frac{\zeta}{k} \frac{\partial}{\partial r}\left(r H_{\varphi}\right),
$$

or in the dimensionless form 

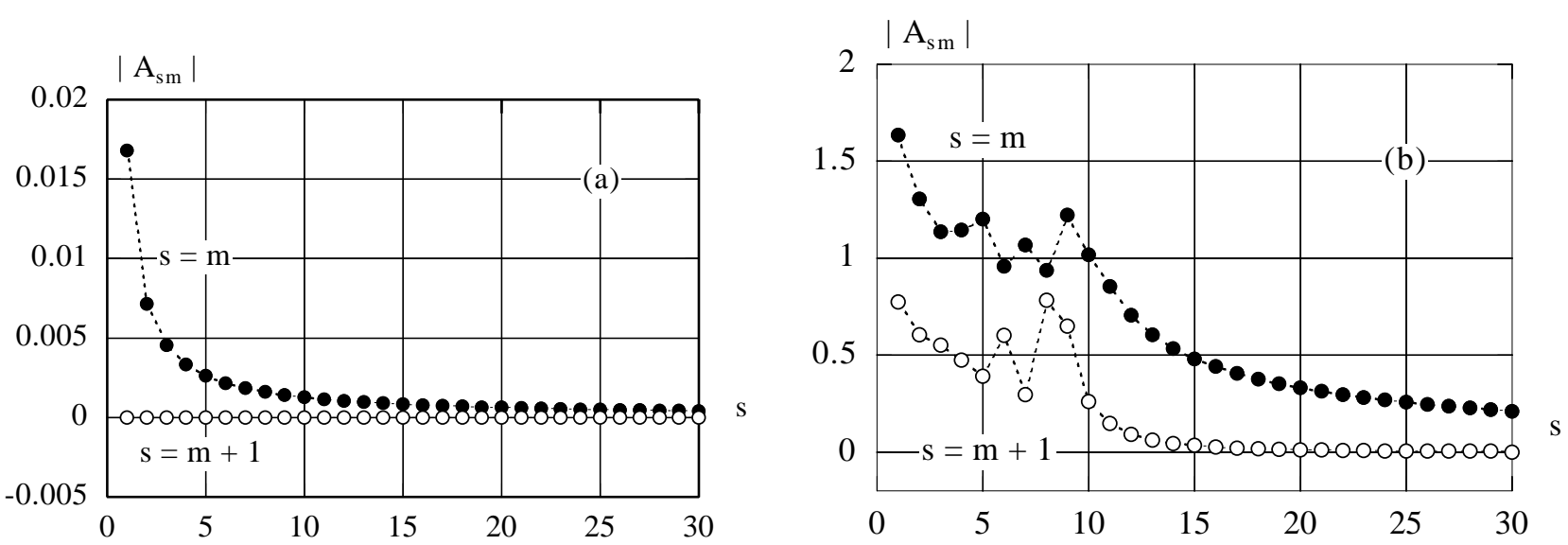

FIG. 3. The coefficient matrix (main diagonal and first parallel) for a small value of $k b[k b=1 / 10]$ (a) and for a large one $[k b=50](\mathrm{b}), w=2 / 5$ and $\beta=1$.

$$
\frac{2 \pi b}{q \zeta}\left[E_{r}(r, z)-E_{r}^{0}(r, z)\right]=\frac{2 \operatorname{sgn}(z)}{j \beta} \sum_{n=1}^{\infty} c_{n} x_{n} \frac{J_{0}\left(x_{n} r / b\right)}{J_{1}\left(x_{n}\right)} \frac{\exp \left[-j|z| \sqrt{(k b)^{2}-x_{n}^{2}}\right]}{\sqrt{(k b)^{2}-x_{n}^{2}}}
$$

and using the relevant result [11]

$$
\begin{array}{r}
\int_{-\infty}^{+\infty} \exp \left[j\left(k z / \beta-|z| \sqrt{(k b)^{2}-x_{n}^{2}}\right)\right] \operatorname{sgn}(z) d z \\
=\frac{2 j}{\beta} \frac{k b^{2}}{(\kappa b)^{2}+x_{n}^{2}},
\end{array}
$$

we can conclude that

$$
\begin{aligned}
Z_{\|}(k) & =R_{\|}(k)+j X_{\|}(k) \\
& =-\frac{2 \zeta}{\pi \beta^{2}} \sum_{n=1}^{\infty} \frac{c_{n}}{(\kappa b)^{2}+x_{n}^{2}} \frac{x_{n}}{J_{1}\left(x_{n}\right)} \frac{k b}{\sqrt{(k b)^{2}-x_{n}^{2}}} .
\end{aligned}
$$

The general expansion (29) simplifies for $\beta=1$, namely $\kappa=0$, as

$$
Z_{\|}(k)=-\frac{2 \zeta}{\pi} \sum_{n=1}^{\infty} \frac{c_{n}}{x_{n} J_{1}\left(x_{n}\right)} \frac{k b}{\sqrt{(k b)^{2}-x_{n}^{2}}} .
$$

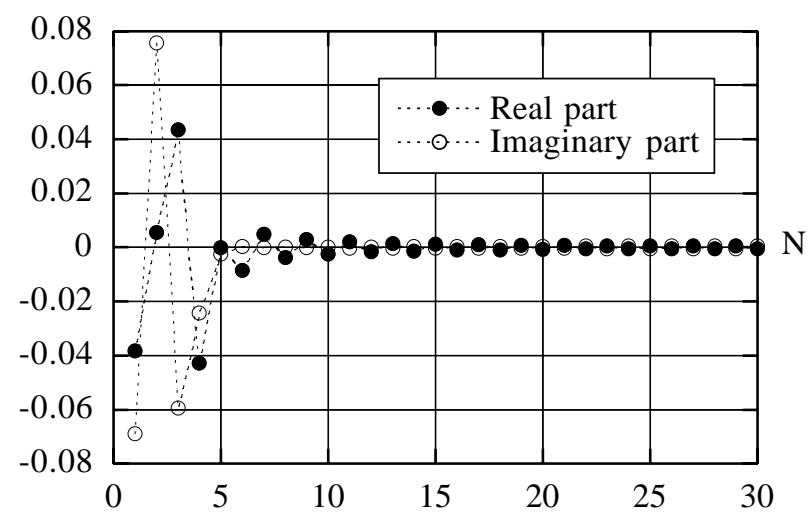

FIG. 4. Expansion coefficients $f_{N}$ versus $N$ in the case $k b=$ $10, w=4 / 5$, and $\beta=1$.
Therefore, a complete solution of the problem of the evaluation of the impedance can be obtained by computing the expansion coefficients $c_{n}$ with a high degree of accuracy.

Figures 5-20 show different impedances versus the frequency (normalized wave number) for different values of the aspect ratio and in a wide range of the speed of the particle.

\section{THE HIGH FREQUENCY LIMIT}

The evaluation of the impedance in the case $\beta=1$ could require a large amount of computing since the frequency spectrum is wider than the other cases. In order to show the accuracy of the numerical evaluation, we verified that our calculations reconstruct the asymptotic behavior of the real part

$R_{\|}(k) \cong \frac{\zeta}{\pi} \ln \frac{b}{a}=-\frac{\zeta}{\pi} \ln w \quad$ when $k b \rightarrow \infty$,

and find another asymptotic expansion valid for all the values of $\beta$.

The dual series system (17) can be formally rewritten as the single equation

$$
\begin{aligned}
\sum_{n=1}^{\infty} c_{n} \alpha_{n}(r) e_{n}(r)= & -\frac{\beta \sqrt{\pi}}{q \zeta} E_{r}^{0}(r, 0) \\
& \times[u(r-a)-u(r-b)], \\
& 0 \leq r \leq b,
\end{aligned}
$$

where the function $\alpha_{n}(r)$ is defined by the relation

$$
\alpha_{n}(r)= \begin{cases}k b / \sqrt{(k b)^{2}-x_{n}^{2}}, & 0 \leq r \leq a, \\ 1, & a \leq r \leq b .\end{cases}
$$


Projecting Eq. (32) on the modes (8) of the waveguide, it becomes

$$
\begin{array}{r}
c_{m}+\sum_{n=1}^{\infty} c_{n} M_{n m}\left[\frac{k b}{\sqrt{(k b)^{2}-x_{n}^{2}}}-1\right]=-b_{m}, \\
m=1,2,3, \ldots,
\end{array}
$$

where we called for shortness [11]

$$
M_{m n}=M_{n m}=\int_{S_{a}} \int e_{m}(r) e_{n}(r) d S,
$$

evaluated in Appendix C. The recurrence equation (33) could be effectively used in a numerical scheme according to field matching analysis, but our interest is to assert that in the high frequency limit, when

$$
\lim _{k b \rightarrow \infty} \frac{k b}{\sqrt{(k b)^{2}-x_{n}^{2}}}=1
$$

$$
\sum_{n=1}^{\infty} \frac{1}{(\kappa b)^{2}+x_{n}^{2}} \frac{J_{0}\left(x_{n} r / b\right)}{J_{1}^{2}\left(x_{n}\right)}=\frac{1}{2}\left[K_{0}(\kappa r)+\frac{K_{0}(\kappa b)}{I_{0}(\kappa b)} I_{0}(\kappa r)\right],
$$

which enables us to find immediately the approximation [11]

$$
\begin{aligned}
\frac{\pi \beta^{2}}{\zeta \kappa^{2}} R_{\|}(k) & \cong \int_{a}^{b} r K_{1}^{2}(\kappa r) d r \\
& =\frac{1}{2}\left\{a^{2}\left[\frac{K_{0}^{2}(\kappa a)-K_{1}^{2}(\kappa a)}{2}\right]-b^{2}\left[\frac{K_{0}^{2}(\kappa b)-K_{1}^{2}(\kappa b)}{2}\right]\right\}-\left[b K_{0}(\kappa b) K_{1}(\kappa b)-a K_{0}(\kappa a) K_{1}(\kappa a)\right] .
\end{aligned}
$$

The approximation (35), valid for all of the values of $\beta$, reduces to the previous (31) in the limit case $\beta=1$.

\section{FINAL REMARKS}

We have discussed a new technique to evaluate the longitudinal coupling impedance of an iris in a circular waveguide.

The problem, formulated as a dual series system, has been reduced to a system of algebraic equations by means of an adequate representation of the unknown expansion the expansion coefficients of the electromagnetic fields are equal and opposite to the coefficients of the primary field, namely $c_{m}=-b_{m}$. Therefore, $Z_{\|}(k) \cong R_{\|}(k)$, namely the imaginary part of the impedance can be neglected, and

$$
\begin{aligned}
R_{\|}(k)= & \frac{2 \zeta}{\pi \beta^{2}} \sum_{n=1}^{\infty} \frac{b_{n}}{(\kappa b)^{2}+x_{n}^{2}} \frac{x_{n}}{J_{1}\left(x_{n}\right)} \\
= & -\frac{2 \zeta \kappa}{\pi \beta^{2}} \int_{a}^{b} r\left[K_{1}(\kappa r)+\frac{K_{0}(\kappa b)}{I_{0}(\kappa b)} I_{1}(\kappa r)\right] \frac{d}{d r} \\
& \times \sum_{n=1}^{\infty} \frac{1}{(\kappa b)^{2}+x_{n}^{2}} \frac{J_{0}\left(x_{n} r / b\right)}{J_{1}\left(x_{n}\right)} d r
\end{aligned}
$$

The series of the previous formula (34) represents a particular case of the Kneser-Sommerfeld formula [1113], given by 


\section{Real part $[\mu \Omega]$}

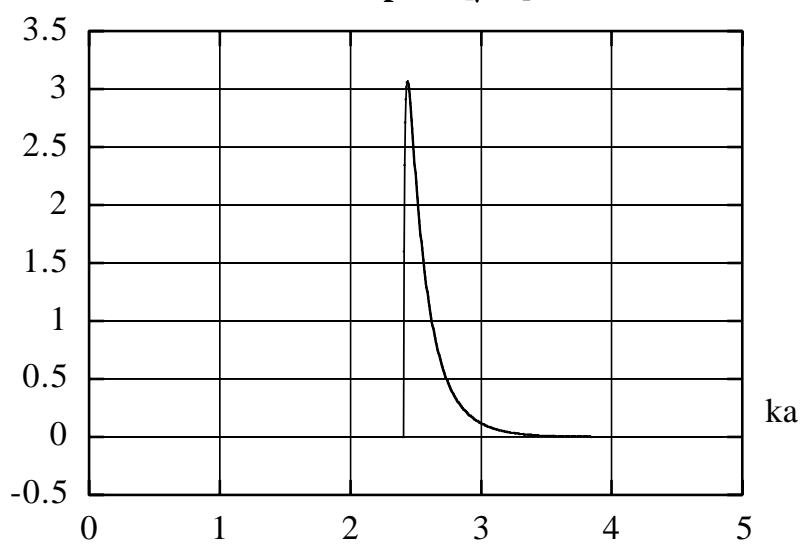

Imaginary part $[\Omega]$

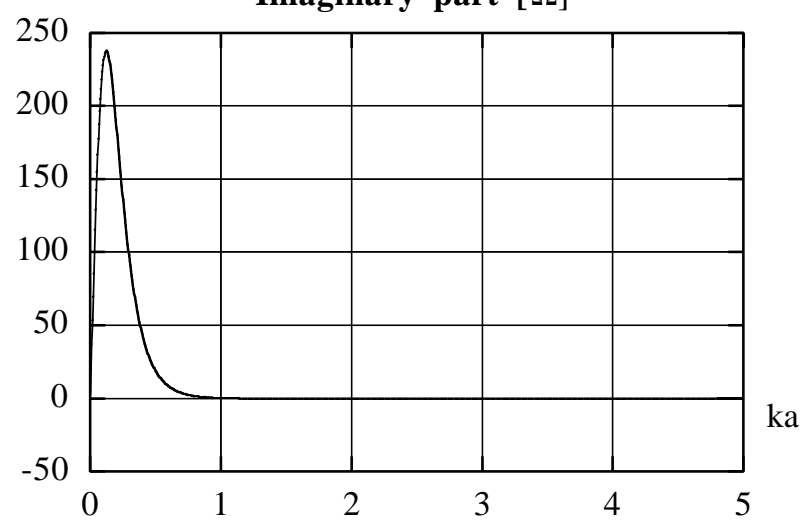

FIG. 6. Real and imaginary parts of the longitudinal coupling impedance in the case $w=2 / 5$ and $\beta \gamma=1 / 10$.
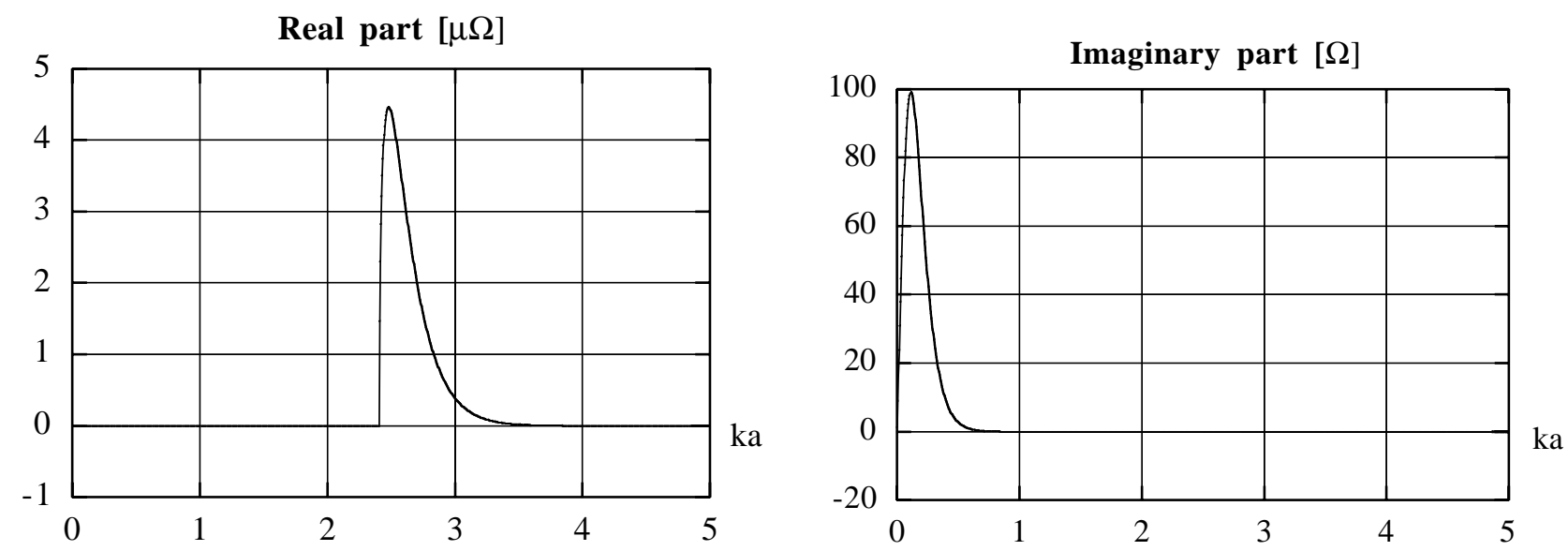

FIG. 7. Real and imaginary parts of the longitudinal coupling impedance in the case $w=3 / 5$ and $\beta \gamma=1 / 10$.
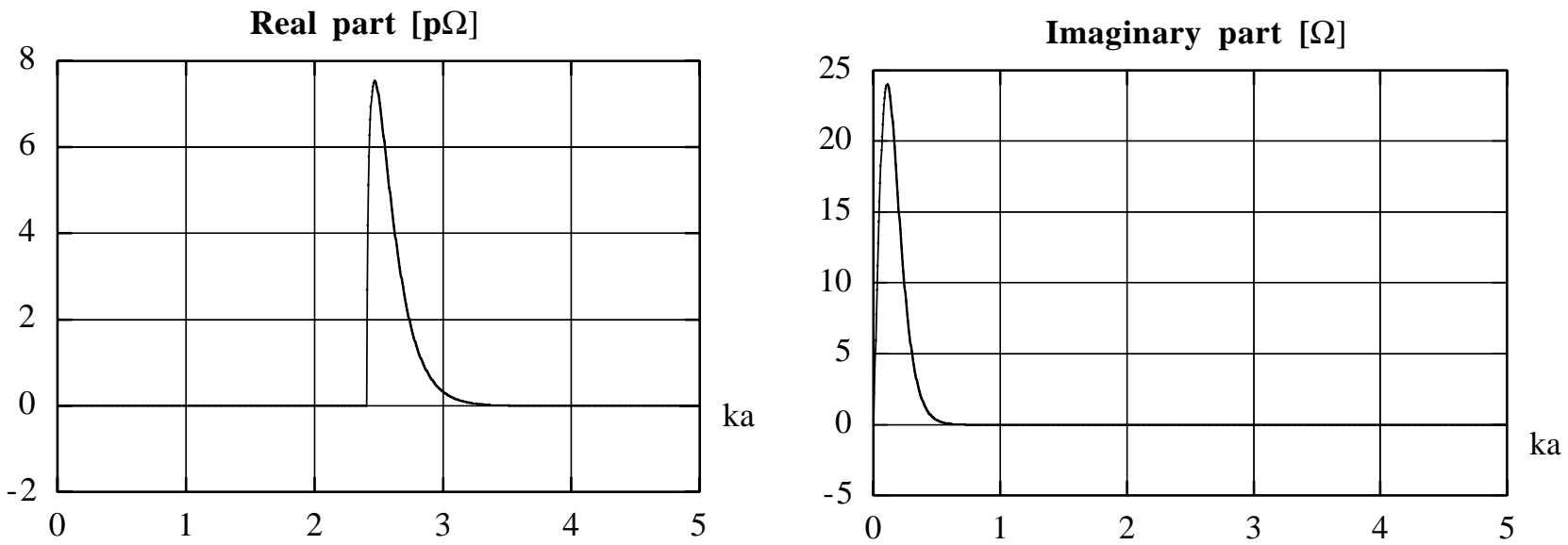

FIG. 8. Real and imaginary parts of the longitudinal coupling impedance in the case $w=4 / 5$ and $\beta \gamma=1 / 10$. 

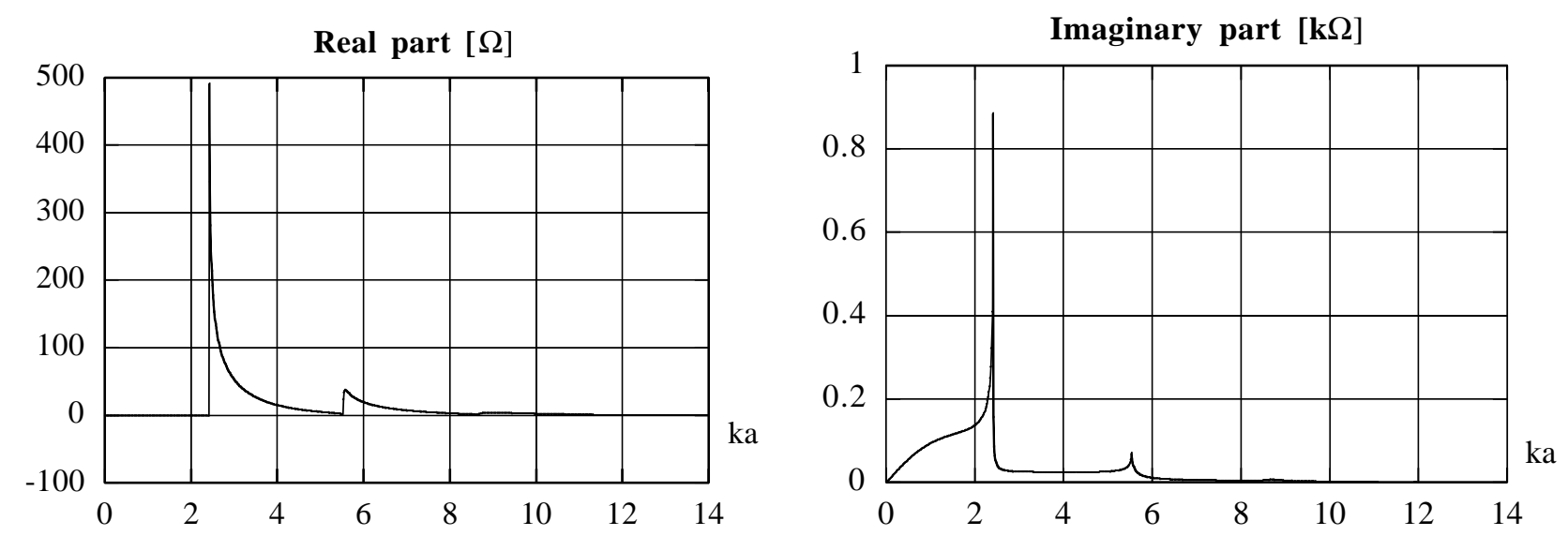

FIG. 9. Real and imaginary parts of the longitudinal coupling impedance in the case $w=1 / 5$ and $\beta \gamma=1$.
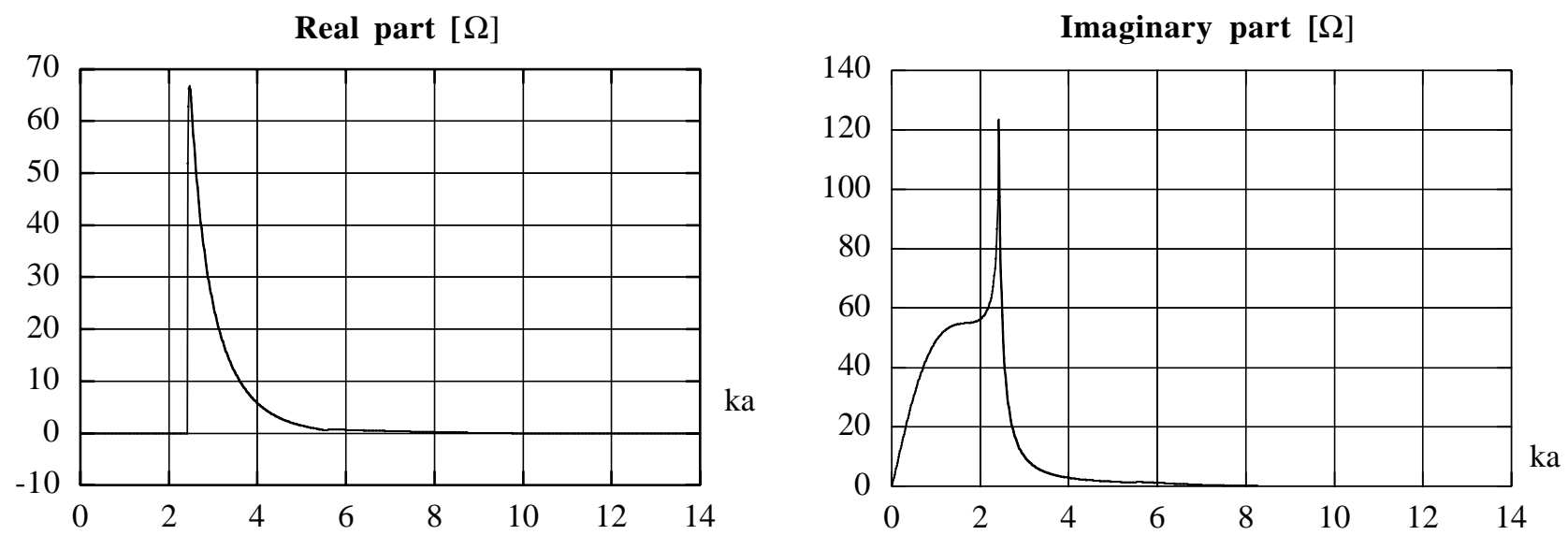

FIG. 10. Real and imaginary parts of the longitudinal coupling impedance in the case $w=2 / 5$ and $\beta \gamma=1$.
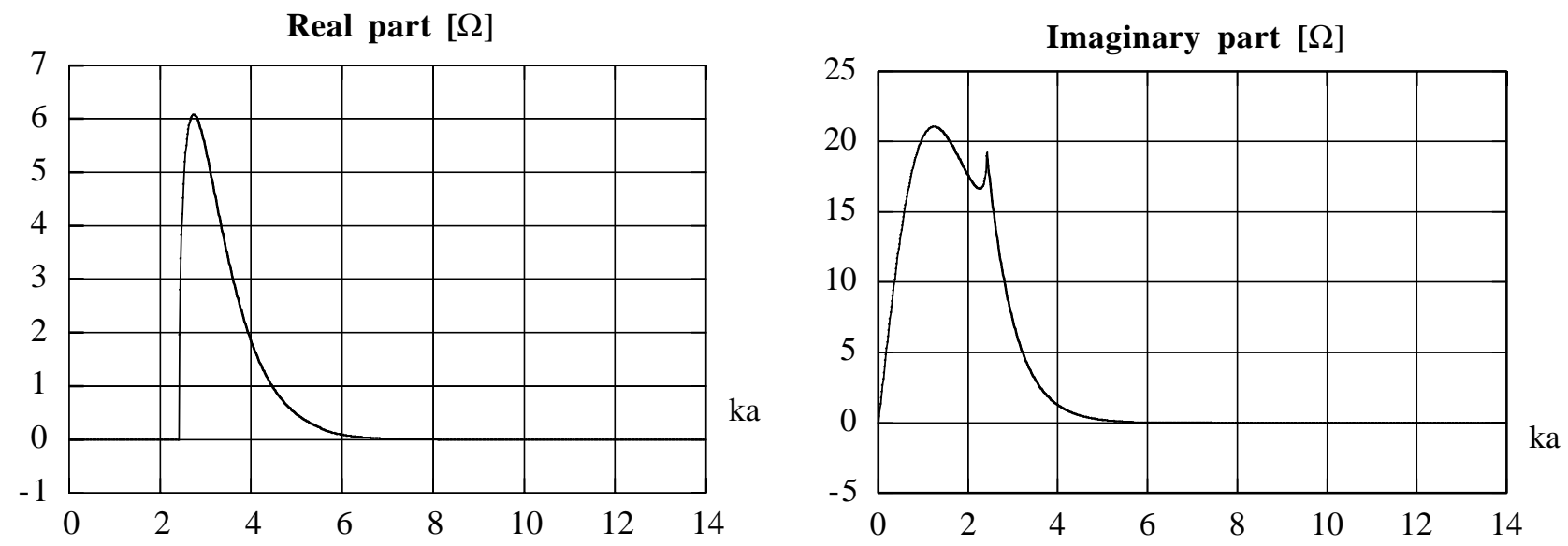

FIG. 11. Real and imaginary parts of the longitudinal coupling impedance in the case $w=3 / 5$ and $\beta \gamma=1$. 

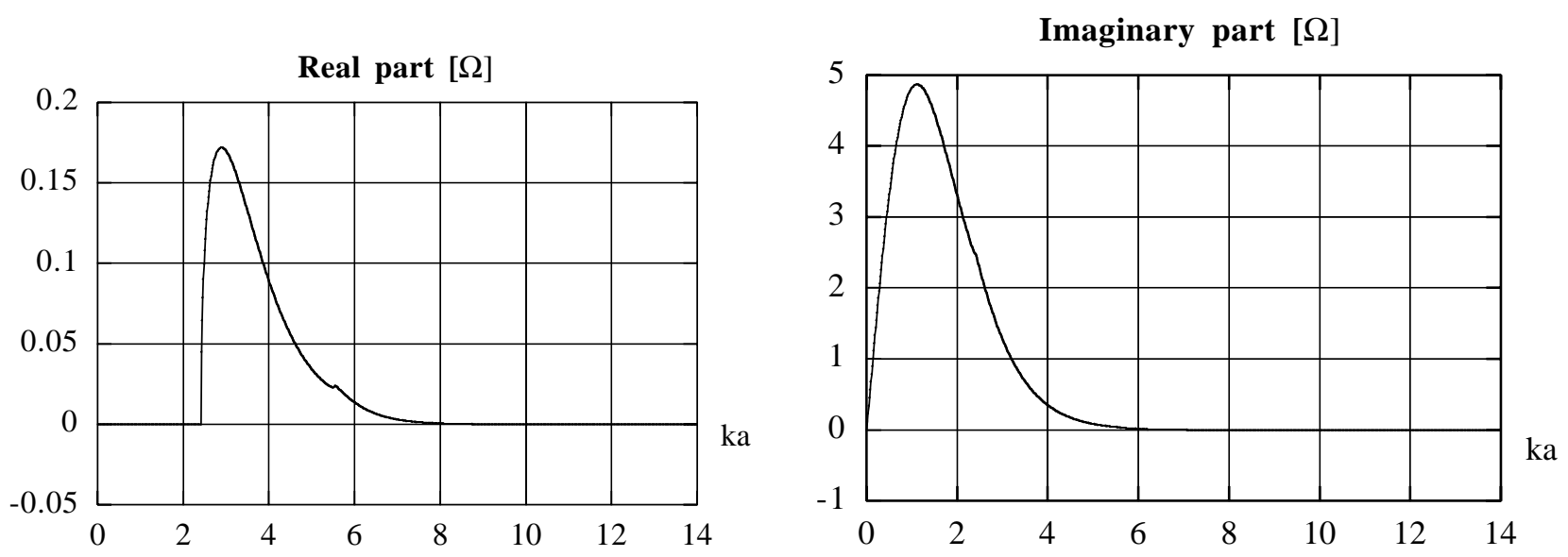

FIG. 12. Real and imaginary parts of the longitudinal coupling impedance in the case $w=4 / 5$ and $\beta \gamma=1$.
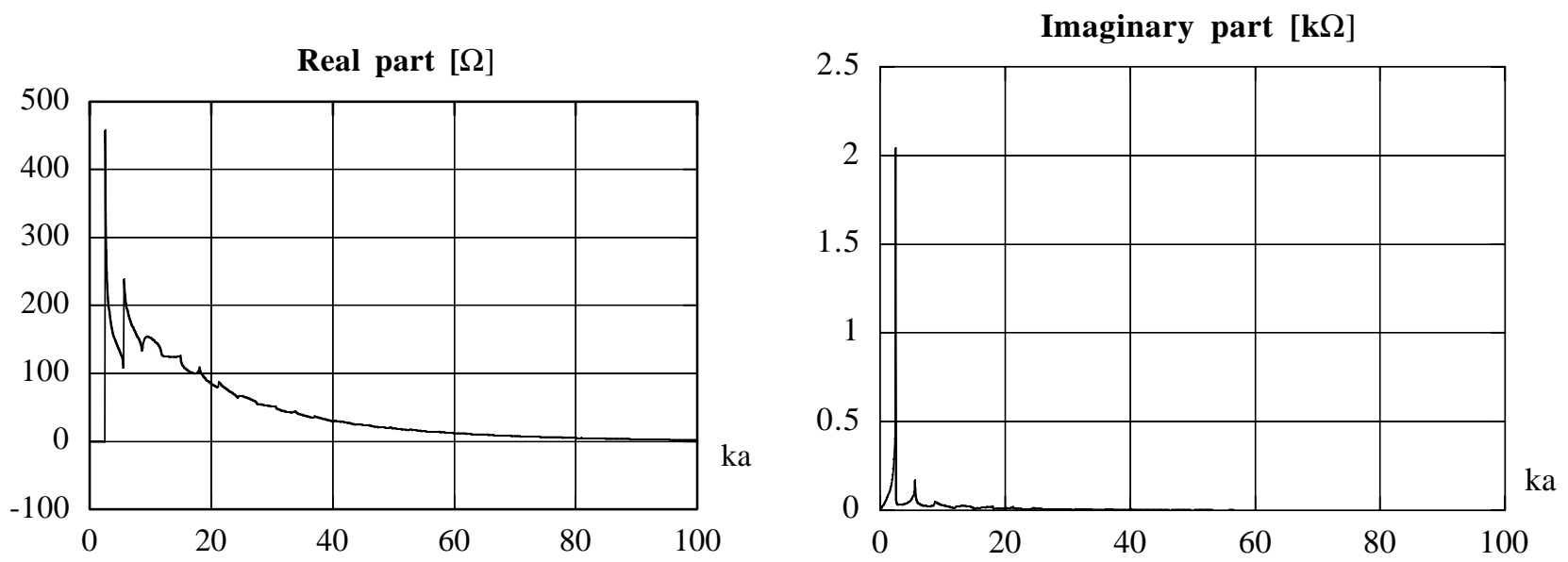

FIG. 13. Real and imaginary parts of the longitudinal coupling impedance in the case $w=1 / 5$ and $\beta \gamma=10$.
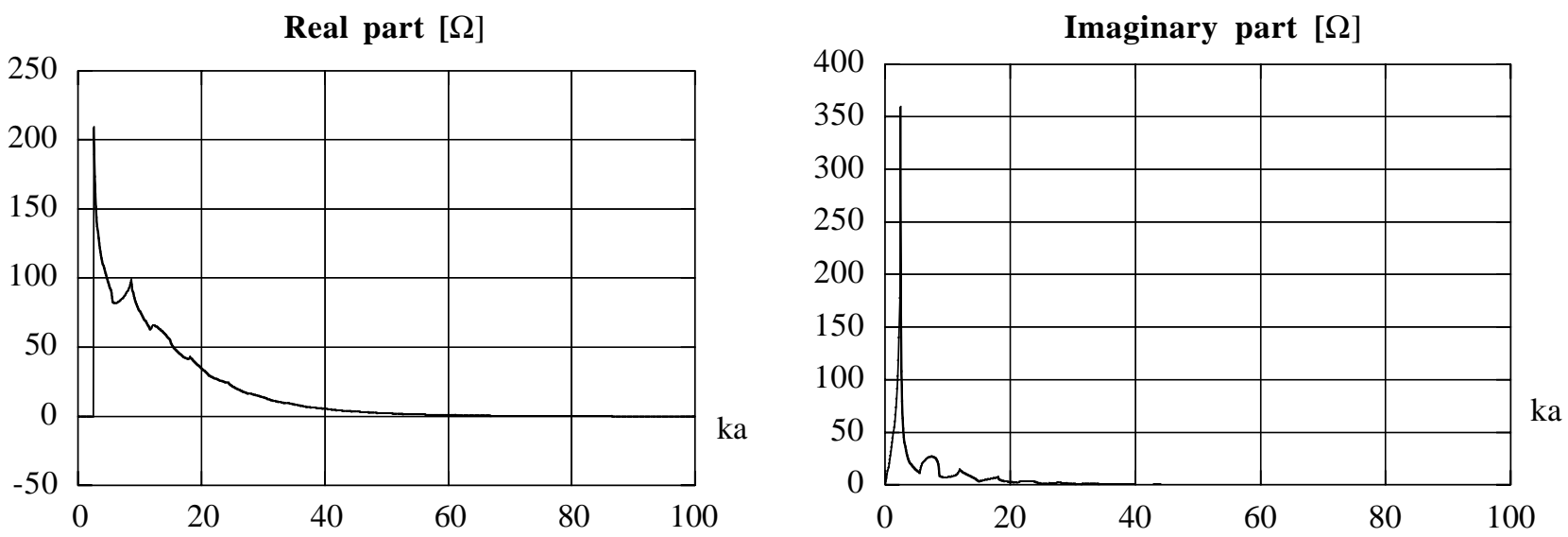

FIG. 14. Real and imaginary parts of the longitudinal coupling impedance in the case $w=2 / 5$ and $\beta \gamma=10$. 

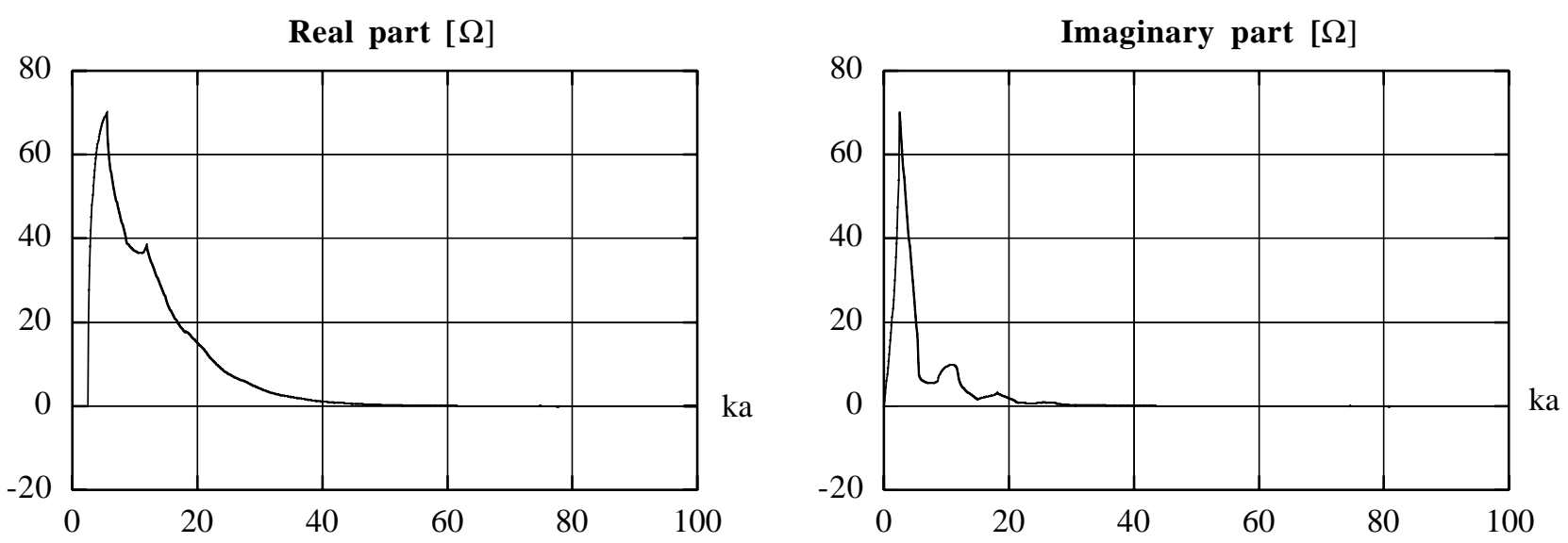

FIG. 15. Real and imaginary parts of the longitudinal coupling impedance in the case $w=3 / 5$ and $\beta \gamma=10$.
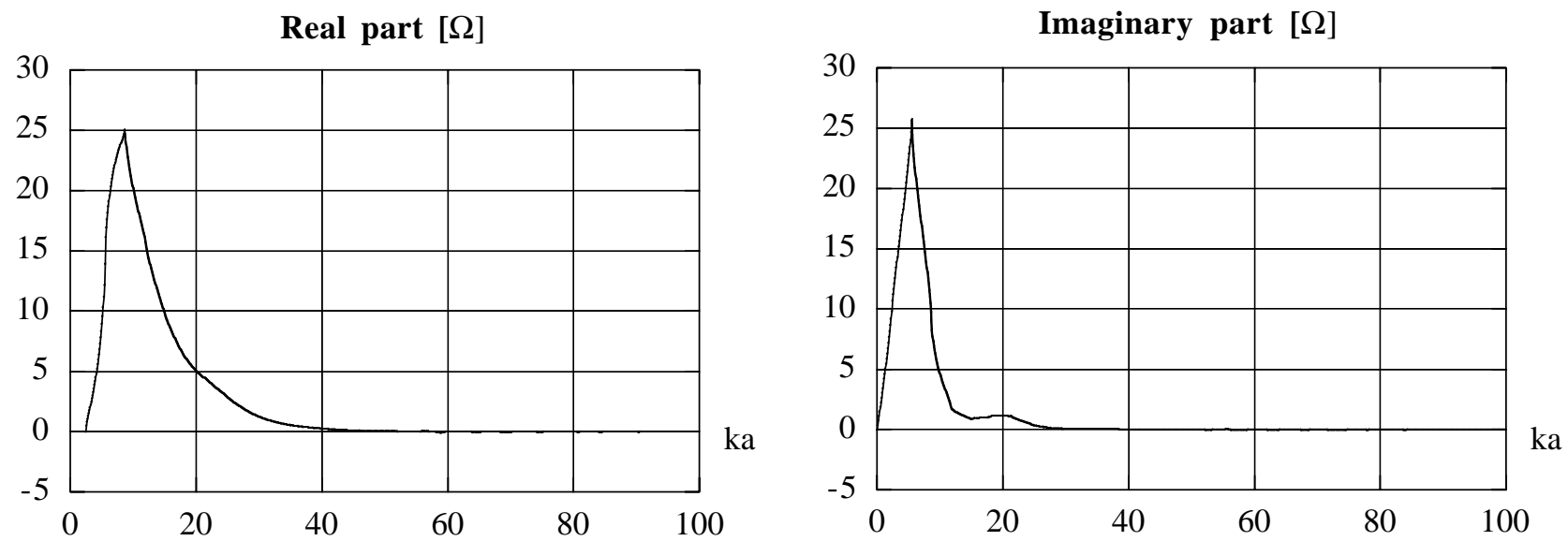

FIG. 16. Real and imaginary parts of the longitudinal coupling impedance in the case $w=4 / 5$ and $\beta \gamma=10$.
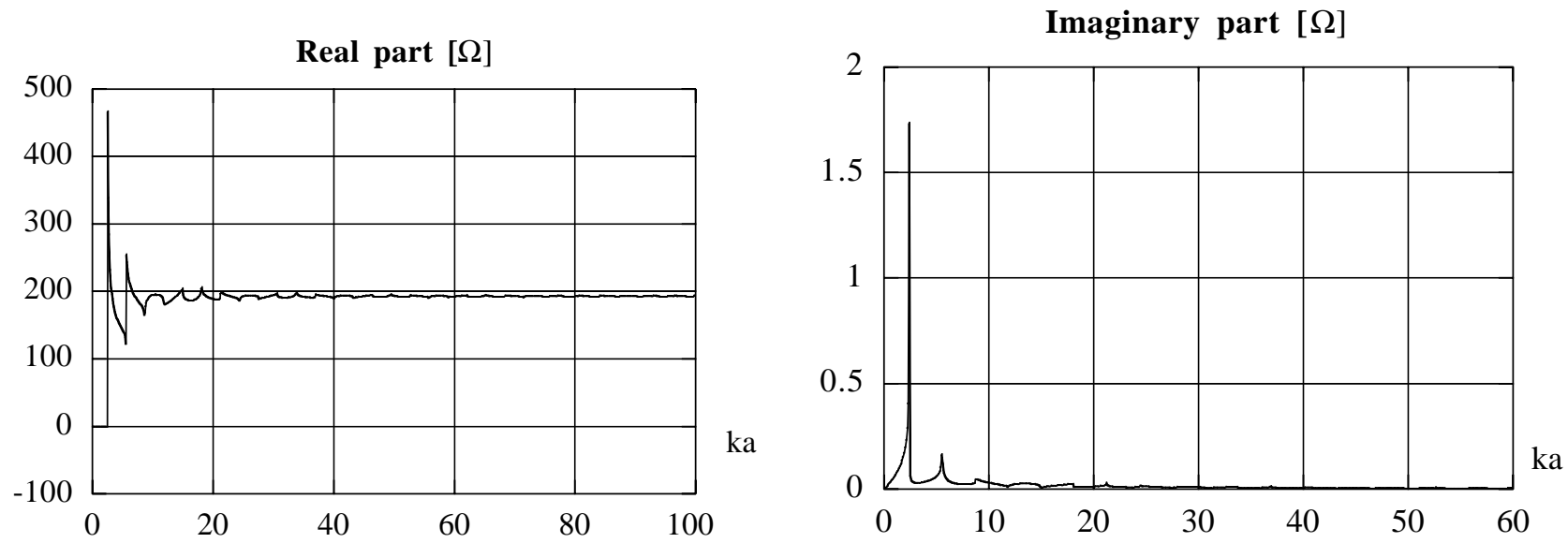

FIG. 17. Real and imaginary parts of the longitudinal coupling impedance in the case $w=1 / 5$ and $\beta=1$. 

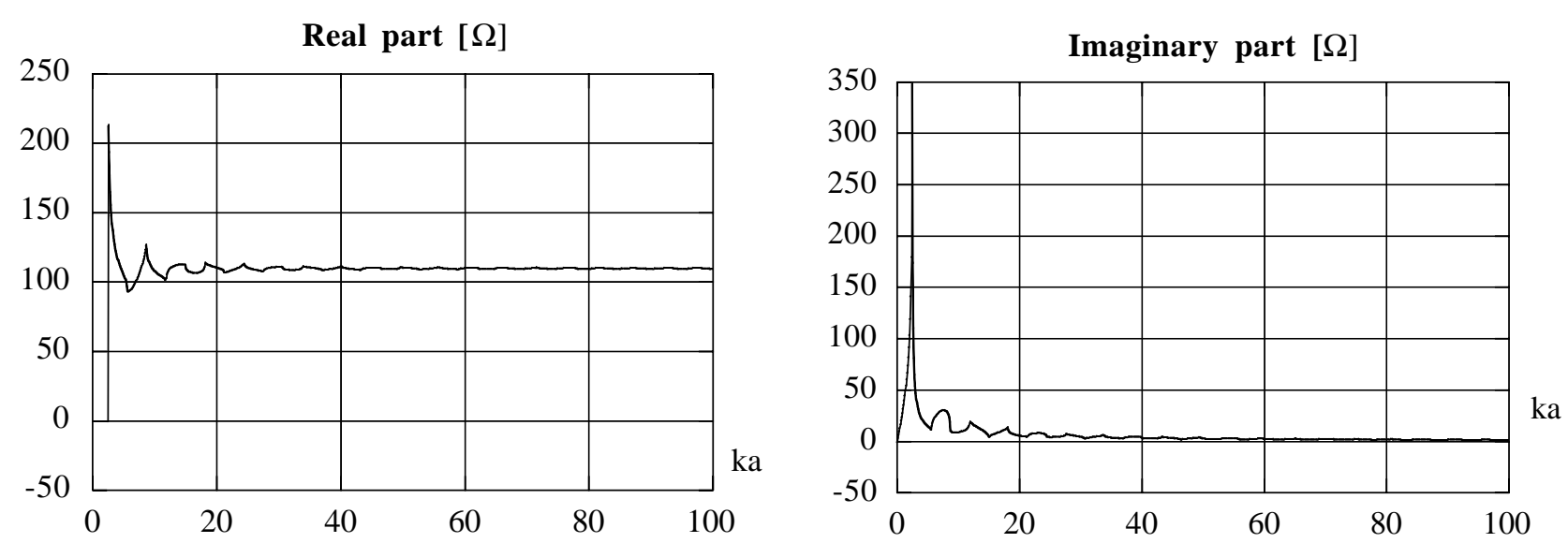

FIG. 18. Real and imaginary parts of the longitudinal coupling impedance in the case $w=2 / 5$ and $\beta=1$.
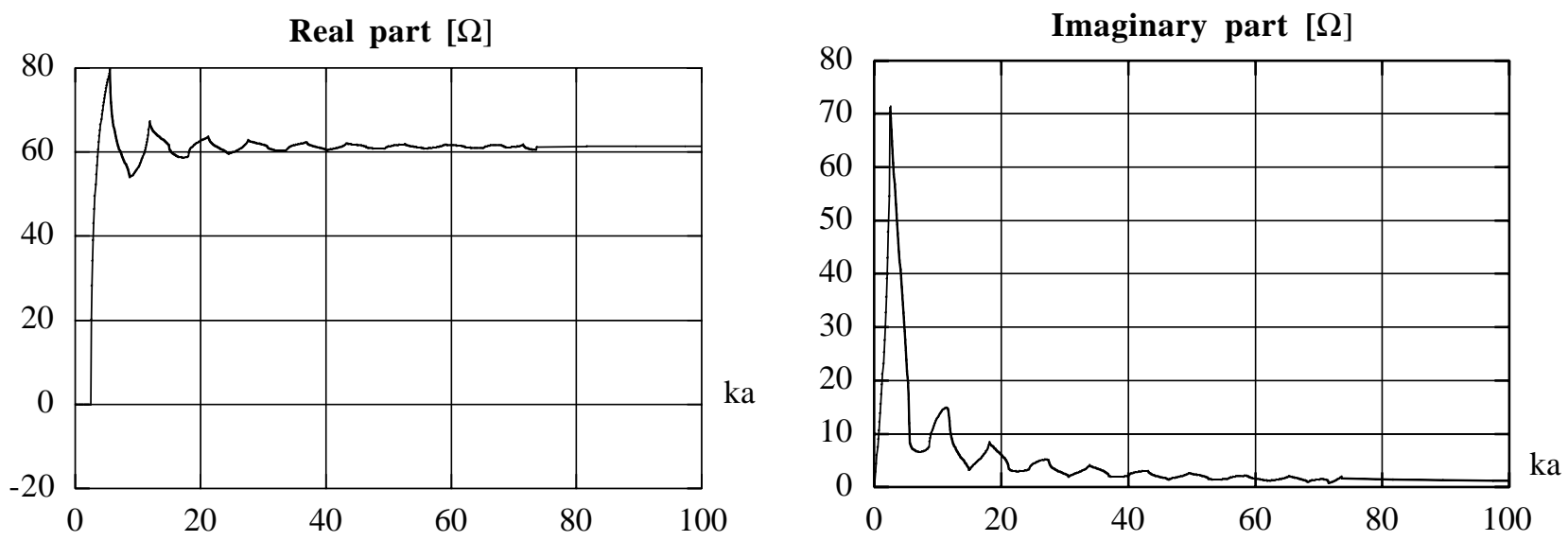

FIG. 19. Real and imaginary parts of the longitudinal coupling impedance in the case $w=3 / 5$ and $\beta=1$.
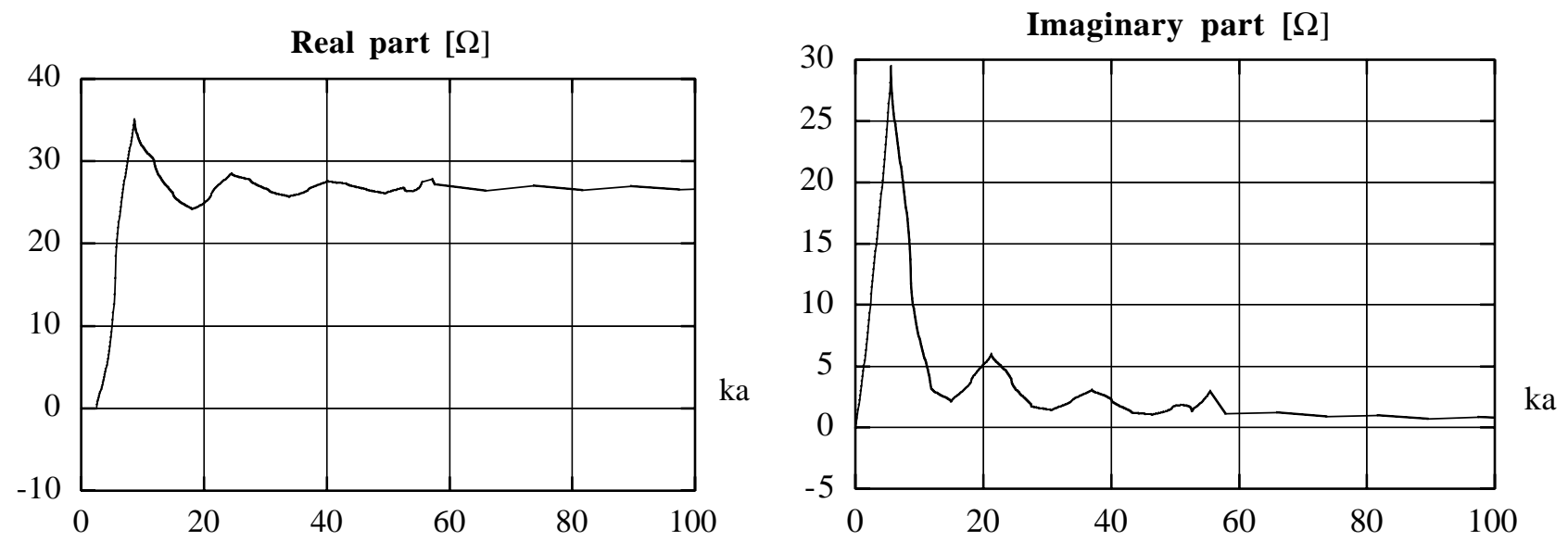

FIG. 20. Real and imaginary parts of the longitudinal coupling impedance in the case $w=4 / 5$ and $\beta=1$. 


\section{ACKNOWLEDGMENT}

The authors are indebted to Professor V. G. Vaccaro of the University of Naples for helpful discussions on this subject.

\section{APPENDIX A}

The aim of this appendix is to evaluate the generic elements $b_{m}$ of the radial component of the primary electric field (18), namely

$$
b_{m}=\frac{\beta \sqrt{\pi}}{q \zeta} \int_{S_{b}} \int_{-S_{a}} E_{r}^{0}(r, 0) e_{m}(r) d S=\frac{\kappa}{b} \int_{a}^{b} r\left[K_{1}(\kappa r)+\frac{K_{0}(\kappa b)}{I_{0}(\kappa b)} I_{1}(\kappa r)\right] \frac{J_{1}\left(x_{m} r / b\right)}{J_{1}\left(x_{m}\right)} d r .
$$

Using the following two integrals involving the product of a Bessel function with a modified one [11],

$$
\begin{gathered}
\int_{0}^{r} u I_{1}(x u) J_{1}(y u) d u=\frac{x r I_{0}(x r) J_{1}(y r)-y r I_{1}(x r) J_{1}(y r)}{x^{2}+y^{2}}, \\
\int_{u}^{r} u K_{1}(x u) J_{1}(y u) d u=\frac{x r K_{0}(x r) J_{1}(y r)+y r K_{1}(x r) J_{0}(y r)}{x^{2}+y^{2}}+\frac{y}{x\left(x^{2}+y^{2}\right)},
\end{gathered}
$$

we can easily evaluate the coefficients

$$
\begin{aligned}
b_{m}= & \kappa a \frac{J_{0}\left(x_{m} w\right)}{J_{1}\left(x_{m}\right)} \frac{x_{m}}{x_{m}^{2}+(\kappa b)^{2}} \frac{K_{1}(\kappa a) I_{0}(\kappa b)+K_{0}(\kappa b) I_{1}(\kappa a)}{I_{0}(\kappa b)} \\
& +\kappa^{2} a b \frac{J_{0}\left(x_{m} w\right)}{J_{1}\left(x_{m}\right)} \frac{1}{x_{m}^{2}+(\kappa b)^{2}} \frac{K_{0}(\kappa a) I_{0}(\kappa b)-K_{0}(\kappa b) I_{0}(\kappa a)}{I_{0}(\kappa b)},
\end{aligned}
$$

which, in the particular case $\beta=1$, can be simplified as

$$
b_{m}=\frac{J_{0}\left(x_{m} w\right)}{x_{m} J_{1}\left(x_{m}\right)}
$$

\section{APPENDIX B}

In order to check the validity of the representation (21), we start with the relevant result [11]

$$
\int_{0}^{\infty} \sqrt{p} J_{2 m-1 / 2}(p) J_{1}(p x) d p=x \sqrt{2} \frac{\Gamma(m+1)}{\Gamma(m-1 / 2)} F\left(m+1,-m+3 / 2 ; 2 ; x^{2}\right) .
$$

Using the property of the hypergeometric function [11] $F(a, b ; c ; z)=(1-z)^{c-a-b} F(c-a, c-b ; c ; z)$, the integral (B1) can be rewritten in terms of the Jacobi polynomials as [11]

$$
F\left(m+1,-m+3 / 2 ; 2 ; x^{2}\right)=\frac{F\left(1-m, m+1 / 2 ; 2 ; x^{2}\right)}{\sqrt{1-x^{2}}}=\frac{P_{m-1}^{(1,-1 / 2)}\left(1-2 x^{2}\right)}{\sqrt{1-x^{2}}} \frac{\Gamma(m)}{\Gamma(m+1)} .
$$

Taking the inverse Hankel transform [5], we get

$$
\frac{J_{2 m-1 / 2}(p)}{\sqrt{p}}=\frac{\sqrt{2} \Gamma(m)}{\Gamma(m-1 / 2)} \int_{0}^{1} x^{2} \frac{P_{m-1}^{(1,-1 / 2)}\left(1-2 x^{2}\right)}{\sqrt{1-x^{2}}} J_{1}(p x) d x .
$$

Thus we get for the representation (21) the expansion coefficients

$$
\begin{aligned}
F_{n m} & =\int_{S_{b}} \int F_{m}(r) e_{n}(r) d S \\
& =\frac{2 \sqrt{2 \pi} \Gamma(m)}{b \Gamma(m-1 / 2)} \int_{0}^{a} r^{2} \frac{P_{m-1}^{(1,-1 / 2)}\left[1-2(r / a)^{2}\right]}{\sqrt{a^{2}-r^{2}}} \frac{J_{1}\left(x_{n} r / b\right)}{J_{1}\left(x_{n}\right)} d r \\
& =2 w \sqrt{\frac{\pi a b}{x_{n}}} \frac{J_{2 m-1 / 2}\left(w x_{n}\right)}{J_{1}\left(x_{n}\right)},
\end{aligned}
$$


and, therefore, the series representation of the class of functions $F_{m}(r)$ enables us to write

$$
\sum_{n=1}^{\infty} \frac{J_{2 m-1 / 2}\left(w x_{n}\right) J_{1}\left(x_{n} r / b\right)}{\sqrt{x_{n}} J_{1}^{2}\left(x_{n}\right)}=\frac{w^{-3 / 2} \Gamma(m)}{\sqrt{2} \Gamma(m-1 / 2)} \frac{r P_{m-1}^{(1,-1 / 2)}\left[1-2(r / a)^{2}\right]}{\sqrt{a^{2}-r^{2}}}, \quad 0 \leq r<a,
$$

or in the complementary region

$$
\sum_{n=1}^{\infty} \frac{J_{2 m-1 / 2}\left(w x_{n}\right) J_{1}\left(x_{n} r / b\right)}{\sqrt{x_{n}} J_{1}^{2}\left(x_{n}\right)}=0, \quad a<r \leq b .
$$

\section{APPENDIX C}

We evaluate in this appendix the generic matrix element $M_{n m}$, defined by the integral

$$
M_{n m}=\int_{S_{a}} \int e_{n}(r) e_{m}(r) d S=M_{m n},
$$

which can be easily computed by means of the relevant and well-known integral involving the product of two Bessel functions [11]

that in the particular case $x=y$ becomes

$$
\int r J_{1}(x r) J_{1}(y r) d r=\frac{y r J_{1}(x r) J_{0}(y r)-x r J_{0}(x r) J_{1}(y r)}{x^{2}-y^{2}},
$$

$$
\int r J_{1}^{2}(x r) d r=\frac{r^{2}}{2}\left[J_{1}^{2}(x r)+J_{0}^{2}(x r)-\frac{2}{x r} J_{0}(x r) J_{1}(y r)\right] .
$$

It is not difficult to show that

$$
M_{n m}= \begin{cases}\frac{2 w}{J_{1}\left(x_{n}\right) J_{1}\left(x_{m}\right)} \frac{x_{m} J_{1}\left(w x_{n}\right) J_{0}\left(w x_{m}\right)-x_{n} J_{0}\left(w x_{n}\right) J_{1}\left(w x_{m}\right)}{x_{n}^{2}-x_{m}^{2}}, & n \neq m, \\ \frac{w^{2}}{J_{1}^{2}\left(x_{n}\right)}\left[J_{1}^{2}\left(w x_{n}\right)+J_{0}^{2}\left(w x_{n}\right)-\frac{2}{w x_{n}} J_{0}\left(w x_{n}\right) J_{1}\left(w x_{n}\right)\right], & n=m,\end{cases}
$$

where the symmetry of the matrix $\mathbf{M}=\left[M_{n m}\right]$ is apparent.

Now, making use of the asymptotic expansions of Bessel functions [11] and related zeros, for large values of $n$ and $m$, the coefficient $M_{n m}$ can be approximated as

$$
M_{n m}= \begin{cases}(-1)^{m+n} \frac{\cos [\pi w(m+n+1 / 2)]}{\pi(m+n+1 / 2)}+(-1)^{m+n} \frac{\cos [\pi w(m-n)]}{\pi(m-n)}, & n \neq m, \\ w+\frac{\sin [\pi w(2 m-1 / 2)]}{\pi(2 m-1 / 2)}, & n=m .\end{cases}
$$

The last approximated relations show that we are dealing with a numerically well-posed problem.

[1] L. Palumbo, V. G. Vaccaro, and M. Zobov, Laboratori Nazionali di Frascati Report No. LNF-94/041, 1994.

[2] S. A. Heifets and S. A. Kheifets, Rev. Mod. Phys. 63(3), 631 (1991).

[3] S.A. Kheifets, IEEE Trans. Microwave Theory Tech. MTT-35, 753 (1987).

[4] P.H. Masterman and J. B. Clarricoats, Proc. IEEE 118, No. 1 (1971).

[5] R. L. Gluckstern, CERN Report No. SL/90-113 1990.

[6] Special issue on the Proceedings of the International Symposium on Heavy Ion Inertial Fusion, edited by S. Atzeni and R. A. Ricci [Nuovo Cimento Soc. Ital. Fis.
106A, No. 12 (1993)].

[7] I. N. Sneddon, in Mixed Boundary Value Problems in Potential Theory (North-Holland, Amsterdam, 1966).

[8] C.J. Tranter, in Bessel Functions with Some Physical Applications (The English University Press, London, 1968).

[9] G. Miano, V. G. Vaccaro, and L. Verolino, J. Math. Phys. 36(8), 4087 (1995).

[10] J. Meixner, IEEE Trans. Antennas Propag. 20, 442 (1972).

[11] A. P. Prudnikov, Yu. A. Brychkov, and O. I. Marichev, in Integrals and Series (Gordon and Breach, New York, 1992), Vol. 2.

[12] G. Franceschetti, in Electromagnetics-Theory, Techniques, and Engineering Paradigms (Plenum, New York and London, 1997).

[13] V. G. Vaccaro and L. Verolino, Nuovo Cimento Soc. Ital. Fis. 113B, 1527 (1998). 